\title{
neuromaps: structural and functional interpretation of brain maps
}

\author{
Ross D. Markello ${ }^{1, \dagger}$, Justine Y. Hansen ${ }^{1, \dagger}$, Zhen-Qi Liu ${ }^{1}$, Vincent Bazinet ${ }^{1}$, Golia \\ Shafiei $^{1}$, Laura E. Suárez ${ }^{1}$, Nadia Blostein ${ }^{2}$, Jakob Seidlitz ${ }^{3}$, Sylvain Baillet ${ }^{1}$, Theodore \\ D. Satterthwaite ${ }^{3}$, M. Mallar Chakravarty ${ }^{2}$, Armin Raznahan ${ }^{4}$, Bratislav Misic ${ }^{1 *}$ \\ ${ }^{1}$ Montréal Neurological Institute, McGill University, Montréal, Canada \\ ${ }^{2}$ Cerebral Imaging Center, Douglas Mental Health University Institute, McGill University, Montréal, Canada \\ ${ }^{3}$ Lifespan Informatics and Neuroimaging Center, Perelman School of Medicine, University of Pennsylvania, PA, USA \\ ${ }^{4}$ Section of Developmental Neurogenomics, National Institute of Mental Health, Bethesda, Maryland, USA \\ ${ }^{\dagger}$ These authors contributed equally to this work
}

\begin{abstract}
Imaging technologies are increasingly used to generate high-resolution reference maps of brain structure and function. Modern scientific discovery relies on making comparisons between new maps (e.g. task activations, group structural differences) and these reference maps. Although recent data sharing initiatives have increased the accessibility of such brain maps, data are often shared in disparate coordinate systems (or "spaces"), precluding systematic and accurate comparisons among them. Here we introduce the neuromaps toolbox, an open-access software package for accessing, transforming, and analyzing structural and functional brain annotations. We implement two registration frameworks to generate high-quality transformations between four standard coordinate systems commonly used in neuroimaging research. The initial release of the toolbox features $>40$ curated reference maps and biological ontologies of the human brain, including maps of gene expression, neurotransmitter receptors, metabolism, neurophysiological oscillations, developmental and evolutionary expansion, functional hierarchy, individual functional variability, and cognitive specialization. Robust quantitative assessment of map-to-map similarity is enabled via a suite of spatial autocorrelation-preserving null models. By combining open-access data with transparent functionality for standardizing and comparing brain maps, the neuromaps software package provides a systematic workflow for comprehensive structural and functional annotation enrichment analysis of the human brain.
\end{abstract}

Keywords: neuroimaging | annotations | atlas | registration | coordinate system | software | connectomics | gradients

\section{INTRODUCTION}

Imaging and recording technologies such as magnetic resonance imaging (MRI), electro- and magnetoencephalography (E/MEG), and positron emission tomography (PET) are used to generate high-resolution maps of the human brain. These maps offer insights into the brain's structural and functional architecture, including grey matter morphometry $[9,17]$, myelination $[13,28,36,88]$, gene expression $[3,33]$, cytoarchitecture [66], metabolism [72], neurotransmitter receptors and transporters $[8,32,55,94]$, laminar differentiation [83], intrinsic dynamics [24, 52, 67] and evolutionary expansion [34, 60, 87, 91]. Such maps are increasingly shared on open-access repositories like NeuroVault [29] or BALSA [77], which, collectively, offer a comprehensive multimodal perspective of the central nervous system. However, these data-sharing platforms are restricted to either surface or volumetric data, and do not integrate standardized analytic workflows.

If researchers generate novel brain maps in their work-such as task fMRI activations or case-control cortical thickness contrasts-how can they interpret them? Ideally there should be a way to systematically compare and contextualize new maps with respect to existing structural and functional annotations, using rigorous

\footnotetext{
*bratislav.misic@mcgill.ca
}

statistical methods. In adjacent fields, such as bioinformatics, there already exist multiple widely-used computational methods for functional profiling and pathway enrichment analysis of gene lists $[6,61]$. A comparable structural and functional enrichment tool for neuroimaging would have to support three specific capabilities: (1) a method for generating high-quality transformations across multiple coordinate systems, (2) a curated repository of brain maps in their native space, and (3) a method for estimating map-to-map similarity that accounts for spatial autocorrelation.

Creating brain maps frequently requires collating and aggregating data from many individuals, a multi-step procedure involving myriad methodological decisions. One critical step in this process, however, is the transformation of individual data to a common coordinate system $[19,74]$. This transformation is often performed to account for anatomical differences between individual subjects prior to group aggregation, and makes derived maps more comparable across datasets. Data collected from MRI are traditionally represented as volumetric images and are therefore commonly transformed to a standard "population" image in volumetric space (e.g., the MNI ICBM 152 template; [22, 49]); however, standardized triangular (i.e., "surface") meshes are increasingly used to represent data as well (e.g., the fsaverage, fsLR, and CIVET surfaces; [20, 21, 23, 76]). Ultimately, how researchers choose to represent their data can have important impacts on their analyses, and use of both vol- 


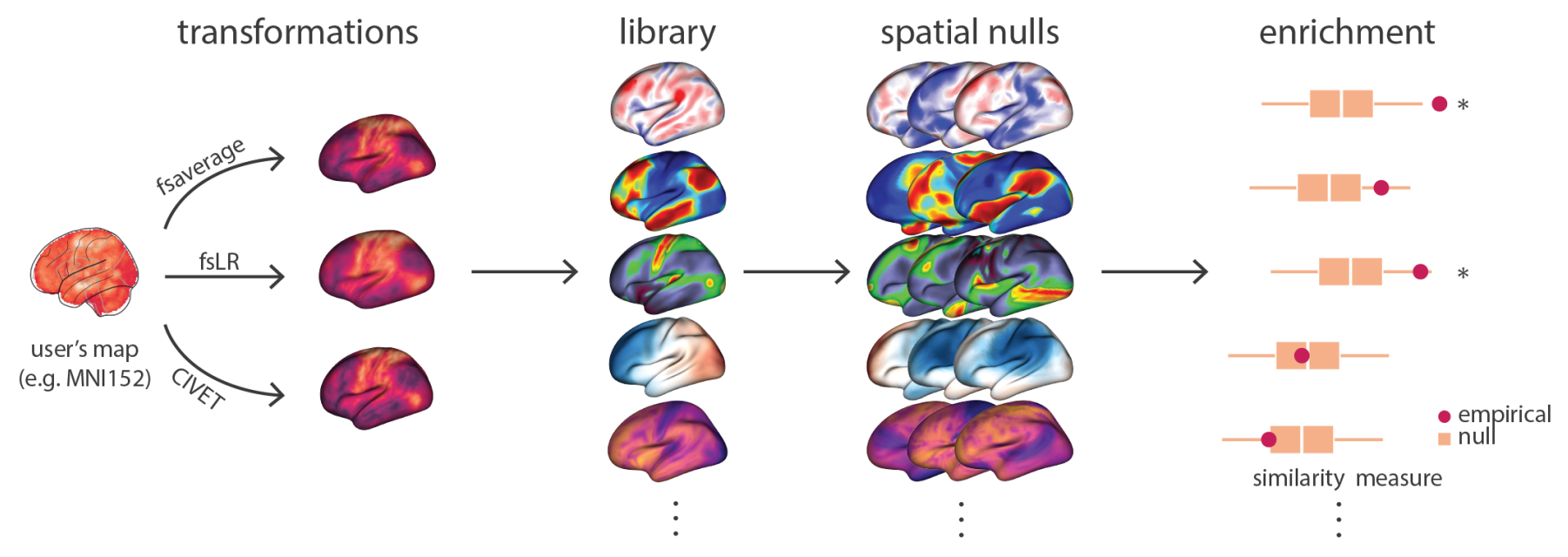

Figure 1. The neuromaps toolbox functionality | The neuromaps software package features a method for generating high-quality transformations across multiple coordinate systems, a curated repository of brain maps in their native systems, and a method for estimating map-to-map similarity that accounts for spatial autocorrelation. For example, user may provide a novel brain map from their own empirical data (e.g. in MNI-152 space), transform it to multiple coordinate systems, and compare it against a library of gradients from the published literature, using spatial autocorrelation preserving null models.

umetric and surface-based coordinate systems remains prevalent in the literature.

Transforming individual, subject-level data between different representations and coordinate systems is nontrivial and has been the focus of significant research over the past several decades $[5,15,30,54,63,64,71,89$, 93]. Comparatively less attention has been given to generating standardized transformations between coordinate systems for group-level or averaged brain maps [42, 43, 57, 90]. However, as researchers continue to produce and share new maps, there is a growing need to both implement robust and accurate group transformations between coordinate systems, and examine whether and how choice of coordinate system may impact comparisons between maps.

In the current report we introduce a new open-access Python toolbox, neuromaps, to enable researchers to systematically share, transform, and compare brain maps (Fig. 1). First, we generate a set of group-level transformations between four standard coordinate systems widely used in neuroimaging and integrate them via a set of accessible, uniform interfaces. Next, we curate over 40 reference brain maps from literature published over the past decade to facilitate contextualizing novel brain annotations. Finally, we implement spatial autocorrelation-preserving null models for statistical comparison between brain maps that will help researchers perform standardized, reproducible analyses of brain maps. Collectively, this represents a first step towards creating systematized knowledge and rapid algorithmic decoding of the multimodal multiscale architecture of the brain.

\section{METHODS AND MATERIALS}

\section{Code and data availability}

All code used for data processing, analysis, and figure generation is available on GitHub (https: //github.com/netneurolab/markello_neuromaps) and directly relies on the following open-source Python packages: BrainSMASH [14], BrainSpace [82], IPython [59], Jupyter [41], Matplotlib [38], NiBabel [11], Nilearn [1], NumPy [56, 73], Pandas [50], PySurfer [86], Scikit-learn [58], SciPy [81], Seaborn [85], and SurfPlot (https://github.com/danjgale/surfplot). Additional software used in the reported analyses includes CIVET (v2.1.1, http://www.bic.mni. mcgill.ca/ServicesSoftware/CIVET [2]), FreeSurfer (v6.0.0, http://surfer.nmr.mgh.harvard.edu/ [21]), and the Connectome Workbench (v1.5.0, https://www.humanconnectome.org/software/ connectome-workbench [45]).

\section{The neuromaps toolbox}

Source code for neuromaps is available on GitHub (https://github.com/netneurolab/neuromaps) and is provided under the Creative Commons AttributionNonCommercial-ShareAlike 4.0 International License (CC-BY-NC-SA; https://creativecommons.org/licenses/ by-nc-sa/4.0/). We have integrated neuromaps with Zenodo, which generates unique digital object identifiers (DOIs) for each new release of the toolbox. Researchers can install neuromaps as a Python package via the PyPi repository (https://pypi.org/project/ neuromaps), and can access comprehensive online 
documentation via GitHub Pages (https://netneurolab. github.io/neuromaps).

\section{Human Connectome Project}

Generating transformations between coordinate systems requires high-quality data from a large cohort of individuals; for the transformations in the neuromaps toolbox we use data from the Human Connectome Project (HCP [78]). Raw T1- and T2-weighted structural MRI data were downloaded for $N=1113$ subjects from the HCP S1200 release. After data processing and omitting subjects that did not successfully complete the CIVET processing pipeline, $N=1045$ subjects remained.

HCP processing pipeline All structural data were preprocessed using the HCP minimal preprocessing pipelines [27, 78]. Briefly, T1- and T2- weighted MR images were corrected for gradient nonlinearity, and when available, images were co-registered and averaged across repeated scans for each individual. Corrected T1w and T2w images were co-registered and cortical surfaces were extracted using FreeSurfer 5.3.0-HCP [17, 20]. Subject-level surfaces were aligned using a multimodal surface matching (MSMAll) procedure [25].

CIVET processing pipeline Images were separately processed with the minc-bpipe-library (https:// github.com/CoBrALab/minc-bpipe-library), which performs N4 bias correction, cropping of the neck region, and brain mask generation. Outputs of minc-bpipe-library were then processed through the CIVET pipeline (v2.1.0 [2]), which performs non-linear registration to the MNI ICBM 152 volumetric template, cortical surface extraction, and registration of subject surface meshes to the MNI ICBM 152 surface template. Due to CIVET processing failures $N=68$ subjects were omitted from further analysis.

\section{Standard coordinate systems}

Here we briefly describe the four standard coordinate systems (one volumetric and three surface) considered in the current report. Although other coordinate systems are used in neuroimaging research, these four arguably represent the most commonly-used systems in the published literature.

\section{The MNI-152 system}

A significant body of work has been dedicated to explaining what is meant when researchers refer to "MNI 152 space", as several variations of this space exist depending on researchers' choice of template [10]. In addition to variations on the MNI-152 template, there exist many other MNI spaces which differ from one another enough to impact downstream analyses [44]. Here we use the MNI-152 space as defined by the template from the Minn/Wash-U Human Connectome Project group [78], which is a variation of MNI ICBM 152 non-linear 6th generation symmetric template (identical to the MNI template provided with the FSL distribution [39]). This template was selected because it is the default template in HCP processing pipelines, of which some were used to generate transformations between coordinate systems. This template was created by averaging the T1w MRI images of 152 healthy young adults that had been linearly and non-linearly (over six iterations) transformed to a symmetric model in Talaraich space.

\section{The fsaverage system}

The fsaverage system, used by FreeSurfer, represents data on the "fsaverage" template, a triangular surface mesh created via the spherical registration of 40 individuals using an energy minimization algorithm to align surface-based features (e.g., convexity; [20, 21]). In current distributions of FreeSurfer there are five scales of the fsaverage template (fsaverage and fsaverage3-6), ranging in density from 642-163,842 vertices per hemisphere. The fsaverage system is roughly aligned to the space of the MNI-305 volumetric system.

\section{The fsLR system}

Proposed by Van Essen et al. [76], the fsLR coordinate system was created to overcome perceived shortcomings of the fsaverage system: namely, hemispheric asymmetry. That is, the left and right hemispheres of the fsaverage surface are not in geographic correspondence, such that vertex $\mathrm{A}$ in the left hemisphere does not correspond to the same brain region as vertex $\mathrm{A}$ in the right hemisphere. Researchers used landmark surface-based registration to align the two hemispheres of the fsaverage surface with a common hybrid target. fsLR templates are available in densities ranging from 32,492-163,842 vertices per hemisphere. The fsLR system is roughly aligned to the space of the MNI-152 volumetric system.

\section{The CIVET system}

The coordinate system used by the CIVET software is a surface reconstruction of the volumetric MNI ICBM 152 non-linear 6th generation template [23]. In its most commonly-used format each hemisphere is represented with 41,962 vertices; a high-resolution version with 163,842 vertices per hemisphere is also available. Because this system is derived from the volumetric MNI template, it ensures that aligned surfaces have good correspondence with volumetric images in the MNI-152 system.

\section{Generating transformations between systems}

Although there are numerous methods for transforming data between coordinate systems, high-quality mappings for group-averaged data are limited [42, 43, 90]. In creating the neuromaps toolbox, we used two 
a | registration fusion

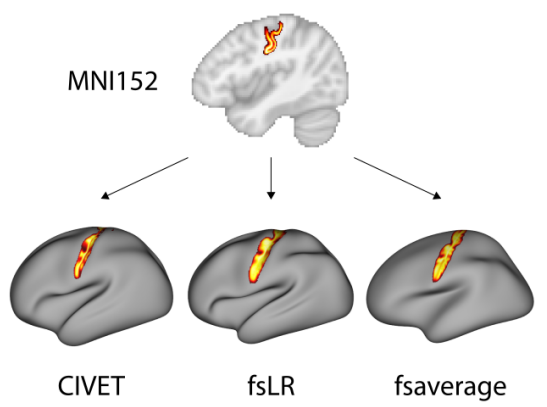

b | multimodal surface matching

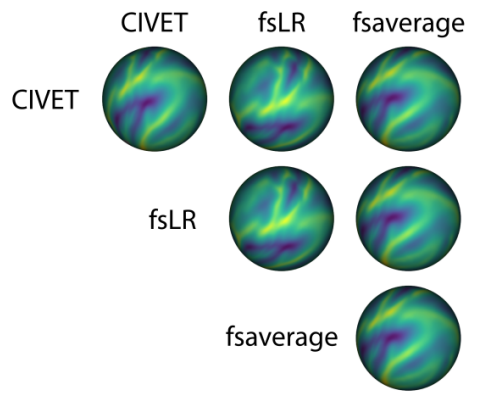

C| one map, many systems (registration fusion)

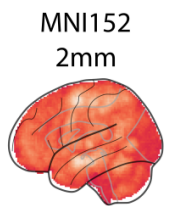

(original)
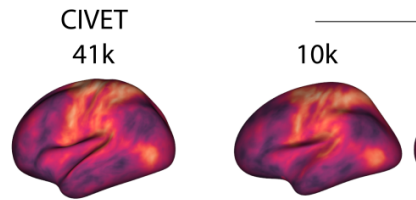

fsaverage

$41 \mathrm{k}$
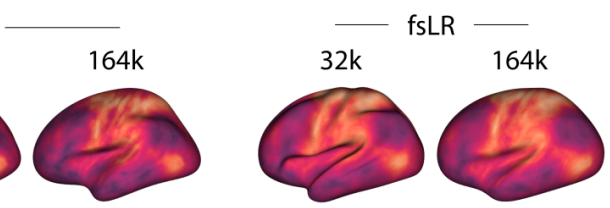

d | one map, many systems (multimodal surface matching)

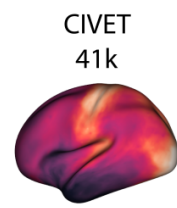

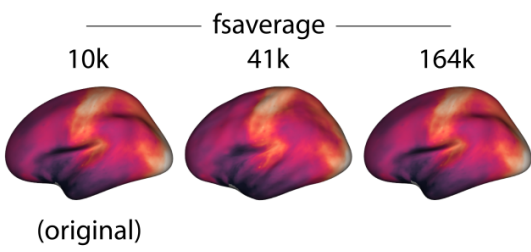

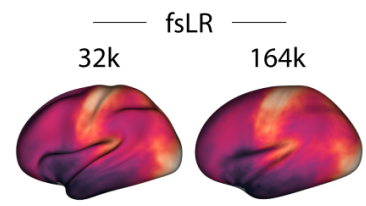

Figure 2. Transformations between coordinate systems | (a) Registration fusion provides a framework for directly projecting group-level volumetric data to the surface. Here, a probabilistic atlas for the central sulcus [90] has been depicted independently on the CIVET (41k), fsLR (32k), and fsaverage (164k) surfaces. (b) Multimodal surface matching (MSM) provides a framework for aligning spherical surface meshes. Here, we show sulcal depth information (originally defined in fsLR space) on spherical meshes that are aligned across the different coordinate systems, where each row represents a different coordinate system and each column represents the space to which that system is aligned. (c) Example of a volumetric brain map (the first principal component of cognitive terms from NeuroSynth [92]) that has been transformed to all surface-based coordinate systems using alignments derived from registration fusion. (d) Example of a surface brain map (the first principal component of gene expression from the Allen Human Brain Atlas [33]) that has been transformed to all other surface-based coordinate systems using alignments derived from MSM. Note that because the original data are represented on the cortical surface, transformation to volumetric space is ill-defined and therefore not shown here.

previously-validated frameworks to generate transformations between all four standard coordinate systems described above (Fig. 2).

\section{Registration fusion framework}

Originally proposed by Buckner et al. [12] and later developed by $\mathrm{Wu}$ et al. [90], registration fusion is a framework for projecting data between volumetric and surface coordinate systems. In its most well-known implementation, researchers used data from the Brain Genomics Superstruct Project (GSP [35]) to generate nonlinear mappings between MNI152 space and the $164 \mathrm{k}$ fsaverage surface [90].

Registration fusion works by generating two sets of mappings for a group of subjects: (1) a mapping between each subject's native image and MNI152 space, and (2) a mapping between each subjects native image and fsaverage space. These mappings are concatenated (MNI152 to native to fsaverage) and then averaged across subjects, yielding a single, high-fidelity mapping that can be applied to new datasets.

Here we generated mappings via registration fusion between the MNI152 volumetric and the fsaverage, fsLR, and CIVET surface-based coordinate systems using data 
microstructure
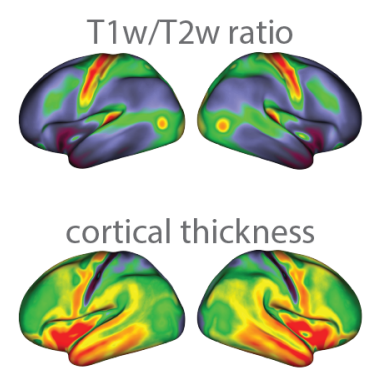

metabolism
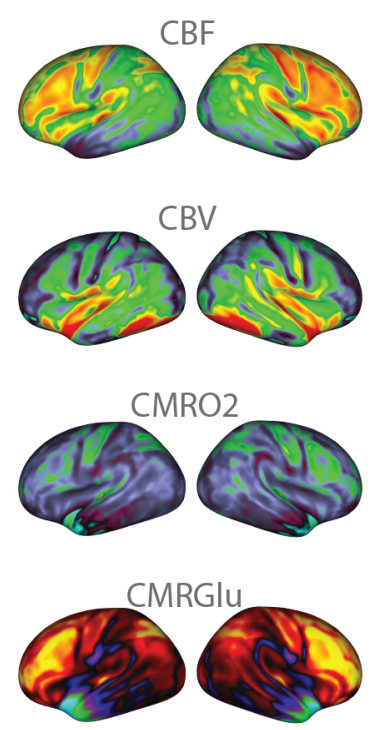

function

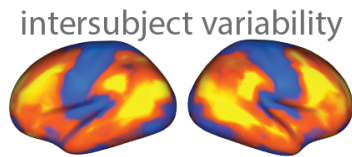

PC1 NeuroSynth

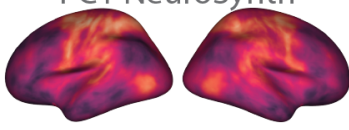

functional gradient

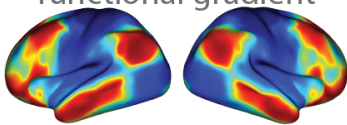

expansion

evo expansion

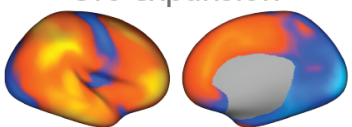

devel expansion

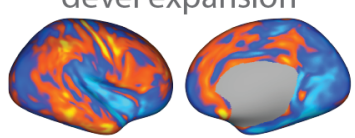

allometric scaling $(\mathrm{NIH})$

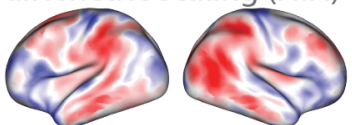

allometric scaling (PNC)

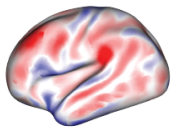

electrophysiology

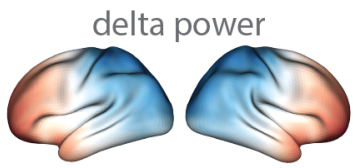

theta power

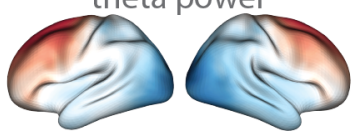

alpha power

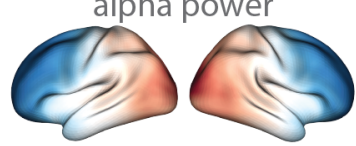

beta power

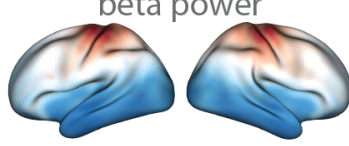

low gamma power

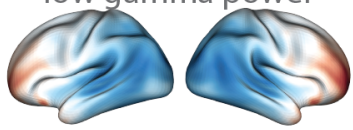

high gamma power

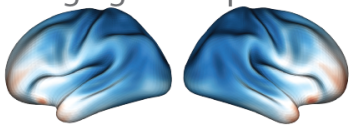

intrinsic timescale

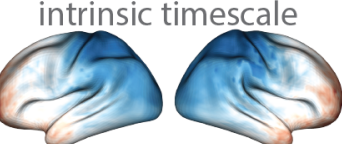

receptors

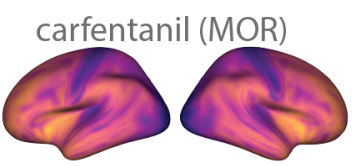

LSN3172176 (M1)

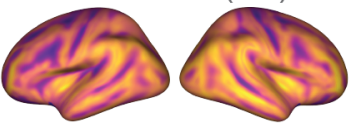

WAY100635 (5-HT1a)

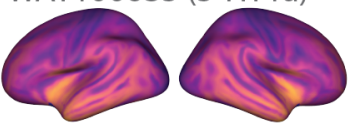

ABP688 (mGluR5)

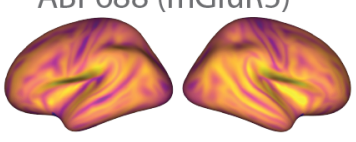

genomics

PC1 gene expression

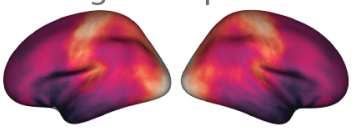

Figure 3. Brain maps from the published literature | Collection of brain maps obtained from the published literature over the past decade that are currently available in the neuromaps distribution. The maps capture the normative multiscale structural and functional organization of the brain, including molecular, cellular, metabolic, and neurophysiological features. Refer to Table S2 for more information on the coordinate system, resolution, and original publication for each brain map. Colormaps were chosen to maximize similarity with how data were represented in the original publication. Note that two of the maps (second column: evo and devel expansion) only have data for the right hemisphere, and the MEG timescale is log-transformed. Additionally, only a selection of four of the 36 neurotransmitter receptor maps are shown here [32, 40, 53, 65, 68]. The repository is continuously updated and the most current list of maps can be found at: https://netneurolab.github.io/neuromaps/. The neuromaps toolbox supports a data contribution pipeline and will continue to expand.

from the HCP (Methods: Data). All mappings used functionality from the Connectome Workbench [45] rather than FreeSurfer to ensure standardization of methodology irrespective of target coordinate systems.

MNI152 to CIVET Unlike for fsaverage and fsLR surfaces, CIVET surfaces are extracted from subject T1 $\mathrm{w}$ volumes after the images have been transformed to the standard MNI152 system. As such, there is no need to generate composite mappings for CIVET surfaces as for the other coordinate systems. Instead, we simply computed the mapping from each subject's MNI152-transformed $\mathrm{T} 1 \mathrm{w}$ volume to the subject's native CIVET surface, and then applied the CIVET-generated surface resampling to register the mapping to the CIVET standard template sys- tem. These mappings were then averaged across subjects to generate a single, group-level transformation.

fsaverage/fsLR/CIVET to MNI152 Although every surface vertex has a corresponding voxel representation in volumetric space, not every voxel has a corresponding vertex representation in surface space. As such, generating transformations from the surface coordinate systems to the MNI152 volumetric system cannot yield a "dense" output map. When Wu et al. [90] proposed the current registration fusion framework they adopted a nearestneighbors, ribbon-filling approach to handle this shortcoming; however, this is only a viable approach when applied to label data (i.e., integer-based parcellation images). We reproduce their approach for completeness, 
but caution against the application of surface-to-volume projections for continuous data and omit such projections from our analyses.

\section{Multimodal surface matching (MSM) framework}

The multimodal surface matching (MSM) framework, initially developed by Robinson et al. [63] and later expanded in Robinson et al. [62], aims to align surfaces defined on different meshes using information from various descriptors of brain structure and function. This procedure has been previously used to generate mappings between the fsaverage and fsLR coordinate systems.

Here, we used MSM to generate a mapping between the CIVET and fsLR systems by aligning HCP subject data processed through the CIVET pipeline with the same data processed through the HCP processing pipeline. As MSM requires input data be provided on spherical surface meshes-a representation not produced in the standard CIVET pipeline-we used FreeSurfer functionality to generate spherical mesh representations and sulcal depth information for each subject's CIVET-derived white matter surfaces. We used these spherical meshes and sulcal depth measurements to drive alignment between the CIVET and fsLR systems via two rounds of the MSM procedure. The first round was used to generate a rotational affine transform to align gross features of the CIVET and fsLR systems; the generated affines were averaged across all subjects and used to seed a second round of finerresolution alignment, similar to the procedure described in Robinson et al. [62]. The final, aligned subject-level spherical surfaces defined in the CIVET system were averaged to create a single, group-level surface that could be used in future transformations.

The CIVET-to-fsaverage mapping was generated as the composite of the transformations between the CIVETand-fsLR and fsLR-and-fsaverage systems.

\section{Parcellations}

Performing analyses at the voxel or vertex level can be computationally intensive. The neuromaps software package can be extended to parcellated data and also integrates easy-to-use tools for parcellating volumetric and surfacic data. The base parcellating function assumes that the given parcellation indexes each region with a unique value, where values of 0 are ignored. However, helper functions are provided to flexibly handle alternative parcellation formats, for example, where both hemispheres are indexed with the same range of values.

\section{Published brain maps}

We curated a selection of brain maps from the published literature of the past decade (Fig. 3). Maps were obtained in their original coordinate system. A complete list of maps and their coordinate systems is provided in Table S2. The neuromaps data repository will continue to evolve and supports a single-function data contribution pipeline for the neuroimaging community to share novel brain annotations in volumetric or surface-based coordinate spaces.

A portion of these maps were originally defined in coordinate systems that are now deprecated. We briefly describe the transformations we used to project these maps to one of the current standard coordinate systems (see Methods: Standard coordinate systems).

PALS-TA24 to fsLR Data obtained from Hill et al. [34] were originally aligned to a study-specific PALS-TA24 template (derived using a similar landmark-based procedure to the PALS-B12 template; [75]), which has been supplanted by the fsLR coordinate system (see Methods: The $f_{s L R}$ system). In order to project data from the PALSTA24 template to the fsLR system we applied the deformation map provided by the original researchers for transforming data between these spaces using nearest neighbors interpolation.

CIVET $v 1$ to $v 2$ The maps obtained from Reardon et al. [60] were originally created using surface templates from CIVET v1.1.12; however, with the release of CIVET v2.0.0 in 2014 the population surface templates provided with the CIVET distribution were updated, effectively deprecating the older templates. In order to project data from the CIVET v1.1.12 templates to the CIVET v2.0.0 templates we used a nearest neighbors interpolation, matching vertex coordinates in the newer template to coordinates in the older template and assigning the value corresponding to the closest vertex [60].

\section{Spatial null frameworks}

Recent research has consistently highlighted the importance of spatially-constrained null models when statistically comparing brain maps [4, 14, 48]. The neuromaps software package integrates nine different spatial null frameworks, described in Markello and Misic [48]. These include six spatial permutation models and three parametrized data models which, collectively, can be constructed for surface-based, volumetric, and parcellated data $[4,7,13,14,16,79,80,82]$. Note that four of the null models are adaptations of the original spatial permutation framework proposed by AlexanderBloch et al. [4] when applied to parcellated data [7, $16,79,80]$. These frameworks differ in how they reassign the medial wall-for which most brain maps contain no data-whether that be by discarding missing data $[7,16]$, ignoring the medial wall entirely [79], or reassigning missing data to the nearest parcel [80]. The three parametrized data models circumvent spatial rotations by applying generative frameworks such as a spatial lag model [13], spectral randomization [82], or variogram matching [14].

For analyses in the current report using surface-based coordinate systems we apply the procedure proposed by Alexander-Bloch et al. [4]; for analyses using volumetric systems we apply the procedure described by Burt 
et al. [14]. Null distributions were systematically derived from 1,000 null maps generated by each framework. The mechanism for each null framework used for analyses in the present work is described briefly below.

\section{Spatial permutation null model}

The procedure proposed by Alexander-Bloch et al. [4] generates spatially-constrained null distributions by applying random rotations to spherical projections of a cortical surface. A rotation matrix $(\mathbf{R})$ is applied to the three-dimensional coordinates of the cortex $(\mathbf{V})$ to generate a set of rotated coordinates $\left(\mathbf{V}_{\text {rot }}=\mathbf{V R}\right)$. The permutation is constructed by replacing the original values at each coordinate with those of the closest rotated coordinate. Rotations are generated independently for one hemisphere and then mirrored across the anteriorposterior axis for the other.

\section{Variogram estimation null model}

The procedure described by Burt et al. [14] operates in two main steps: first the values in a given image are randomly permuted, then the permuted values are smoothed and re-scaled to reintroduce spatial autocorrelation characteristic of the original, non-permuted data. Reintroduction of spatial autocorrelation onto the permuted data is achieved via the transformation $\mathbf{y}=|\beta|^{1 / 2} \mathbf{x}^{\prime}+|\alpha|^{1 / 2} \mathbf{z}$, where $\mathbf{x}^{\prime}$ is the permuted data, $\mathbf{z} \sim \mathcal{N}(0,1)$ is a vector of random Gaussian noise, and $\alpha$ and $\beta$ are estimated via a least-squares optimization between variograms of the original and permuted data.

\section{Assessing the impact of coordinate system}

When transforming two datasets (i.e., a "source" and "target" dataset) defined in distinct coordinate spaces to a common system there are at least three options available: (1) transform the source dataset to the system of the target, (2) transform the target dataset to the system of source, or (3) transform both source and target datasets to an alternate system. If comparisons are being made across several pairs of datasets a fourth option becomes available: (4) always transform the higher resolution dataset to the system of the lower resolution dataset.

To examine whether the choice of coordinate system impacts statistical relationships estimated between brain maps we performed several analyses. First, we transformed a selection of twenty brain maps (see Methods: Published brain maps) into every other coordinate system (e.g., fsaverage $\rightarrow$ fsLR/CIVET/MNI152, fsLR $\rightarrow$ fsaverage/CIVET/MNI152, and so on). We then correlated every pair of these brain maps according to each of the four possible resampling options described above. When transforming both source and target datasets to an alternate system (option 3, above), we comprehensively tested every target coordinate system and data resolution. Spatial null models were used to assess the signifi- cance of all correlations.

\section{RESULTS}

In this report we introduce the neuromaps toolbox, an open-access software package designed to streamline and standardize analyses of neuroimagingderived brain maps (available at https://github.com/ netneurolab/neuromaps). The neuromaps toolbox provides a uniform interface for transforming brain maps between coordinate systems, contextualizing novel brain maps with respect to canonical structural and functional annotations, and assessing relationships between maps using spatial null models. In the following section we highlight features available in neuromaps, demonstrate typical workflows enabled by its functionality, and use neuromaps to examine how choice of coordinate system can impact statistical analyses of brain maps.

The neuromaps data repository

The neuromaps toolbox provides programmatic access to templates for four standard coordinate systems: fsaverage, fsLR, CIVET, and MNI-152 (see Methods: Standard coordinate systems). For surface-based coordinate systems, we distribute template geometry files, sulcal depth maps, and average vertex area shape files (computed from HCP participants) in standard GIFTI format. For volumetric coordinate systems, we distribute T1-, T2-, and PD-weighted template files, a brain mask, and probabilistic segmentations of gray matter, white matter, and cerebrospinal fluid in standard gzipped NIFTI format.

Beyond template files, the neuromaps toolbox offers access to a repository of brain maps obtained from the published literature (Fig. 3; Table S2). These maps were generated using multiple imaging techniques, including magnetic resonance imaging, magnetoencephalography, positron emission tomography and microarray gene expression. Brain maps are provided in the original coordinate system in which they were defined to avoid errors caused by successive interpolation. Collectively, these maps represent more than a decade of human brain mapping research and encompass phenotypes including: the first principal component of gene expression [33], 36 neurotransmitter receptor PET tracer images [32], glucose and oxygen metabolism [72], cerebral blood flow and volume [72], cortical thickness [78], T1w/T2w ratio [25], 6 canonical MEG frequency bands [70, 78], intrinsic timescale [70, 78], evolutionary expansion [34], 3 maps of developmental expansion [34, 60], the first 10 gradients of functional connectivity [46], intersubject variability [51], and the first principal component of Neurosynth-derived cognitive activation [92]. This repository of data is organized by tags and can be easily downloaded directly from neuromaps.

The neuromaps toolbox makes it easy to contextualize novel brain maps to a range of molecular, structural, temporal, and functional features. This will fa- 
a | brain map correlations
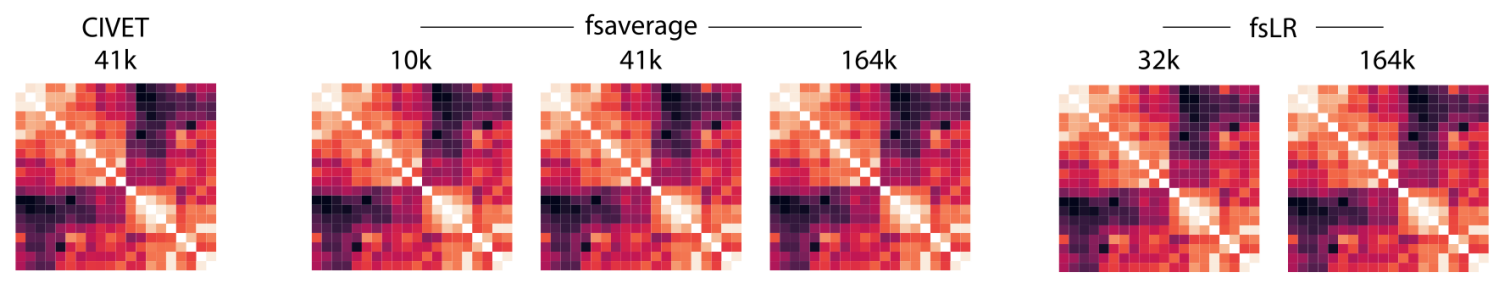

b | embedded network

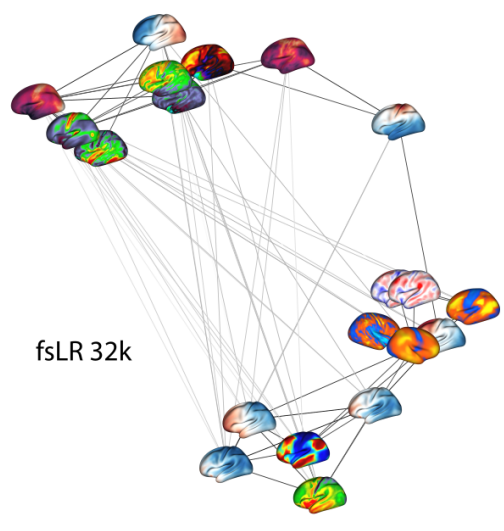

c| map assocations

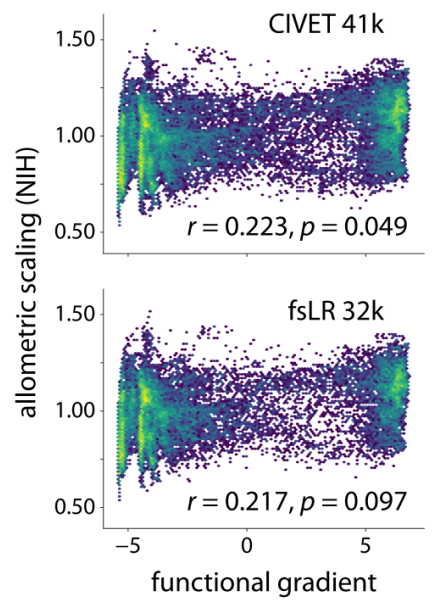

Figure 4. Correlations between brain maps across systems | (a) Correlation matrices between a selection of twenty brain maps in the neuromaps toolbox for each of the surface-based coordinate systems. Because transformations from surface-based to volumetric systems are ill-defined for continuous data we omit those associations. (b) A spring-embedded representation of the correlation matrix among the twenty brain maps, shown here for the fsLR 32k system. (c) An example of two brain maps-the principal functional gradient from Margulies et al. [46] and allometric scaling from Reardon et al. [60] - whose association is significant in one system (CIVET 41k; $r=0.223, p=0.049$ ) and not in another (fsLR 32k; $r=0.217, p=0.097$ ).

cilitate an expansion of research questions, allowing researchers to bridge brain topographies across several spatial scales and across disciplines outside of their immediate scope. Additionally, annotations will be regularly added to the repository, resulting in an increasingly rich toolbox. The neuromaps toolbox also integrates an easy-to-use workflow for researchers who wish to share new brain maps in any of the four supported coordinate systems; information on contributing new brain maps can be found in the online documentation for the software (https://netneurolab.github.io/neuromaps/).

\section{Transformations between coordinate systems}

Despite the multiscale, multimodal collection of brain phenotypes in neuromaps, data cannot be readily compared to one another because they exist in different native coordinate systems. Indeed, a common challenge when relating novel neuroimaging data to the broader literature is finding a common coordinate space or parcellation in which to conduct the analyses. The neuromaps module provides transformations between four supported coordinates systems as well as a standardized set of functions for their application. Transformation between volumetric- and surface-based coordinate systems were derived with a registration fusion framework (Fig. 2a; [90]), whereas transformations between surface-based coordinate systems were derived using a multimodal surface matching (MSM) framework (Fig. 2b; [62, 63]). We leverage tools from the Connectome Workbench to provide functionality for applying transformations between surface systems; however, users do not need to interact directly with these Workbench commands. In addition to transforming individual annotations, the neuromaps software package includes functionalities that resample images to each others' spaces. By default, data is only ever downsampled, which ensures neuromaps does not artificially create new data. Collectively, the neuromaps toolbox facilitates robust transformations between coordinate systems, facilitating the standardization of neuroimaging workflows. 


\section{Assessing relationships between brain maps}

The primary goal for transforming maps to a common coordinate system is to statistically compare their spatial topographies. The neuromaps software package uses a flexible framework for examining relationships between brain maps, offering researchers the ability to provide their own image similarity metric or function, as well as easy handling of missing data. By default, the primary map comparison workflows use the standard Pearson correlation to test the association between provided maps. The neuromaps comparison workflow also integrates multiple methods of performing spatial permutations for significance testing.

Multiple spatial null model frameworks allow for statistical comparison between brain maps while accounting for spatial autocorrelation $[4,7,13,14,16,79,80$, 82]; however, the implementation of these models varies and, to-date, there has been limited effort to provide a standardized interface for their use. We have incorporated nine null models into the neuromaps toolbox, offering a common user interface for each model that can be easily integrated with other aspects of the toolbox. Given the computational overhead of these models, our implementations offer mechanisms for caching intermediate results to enable faster re-use across multiple analyses. Spatial null models are enabled by default in the primary map comparison workflows to encourage their broader adoption. Based on prior work benchmarking the accuracy and computational efficiency of these models [48] we set the non-parametric method originally described in Alexander-Bloch et al. [4] as the default for use with surface data, and the parameterized generative method described in Burt et al. [14] as the default for use with volumetric data.

To demonstrate the utility of neuromaps, we analyzed a sample of 20 brain maps from the published literature over the past decade (2011-2021), including two microstructural, four metabolic, three functional, four expansion, six band-specific electrophysiological signal power, and one genomic annotation. We then used neuromaps to transform these maps from their original representation to the space defined by each of four standard coordinate systems, for a total of seven different representations (Fig. 3). Finally, we computed the pairwise correlations between all maps in each of the systems and assessed the statistical significance of these relationships using spatial null models (see Methods: Spatial null frameworks). The goal of this analysis was twofold. First, we sought to assess the extent to which coordinate transforms could influence map-to-map comparisons. Second, given the growing interest in how these system-level maps or "gradients" are related to one another, we sought to assess patterns of relationships among them $[37,69]$.

Fig. 4a shows that for most map-to-map comparisons, choice of coordinate system has a minimal effect: correlations between maps on average only change by $|r|=$
0.018. There are also instances in which associations between maps are statistically significant in one coordinate system and not significant in another (Fig. 4c); however, in most cases the p-value for these relationships often fell very close to the statistical alpha (i.e., $p \leq 0.05$ ) such that the actual effect size only changed by $r \leq 0.10$. Across all examined systems, we find that the brain maps tend to form two distinct clusters (Fig. 4b), largely recapitulating previously-established relationships observing anterior-posterior and unimodal-transmodal axes of variation [31, 46, 67]. These results are encouraging, suggesting that transforming brain annotations between different systems generally preserves their relationships.

\section{DISCUSSION}

Neuroimaging researchers are constantly generating new datasets that offer unique perspectives into the structural and functional architecture of the human brain. Contextualizing new datasets with multiscale, multimodal brain organization requires both a curated repository of annotations and transparent functionality for transforming and comparing brain maps. Here we introduced the neuromaps toolbox which offers both a repository of widely-used brain map datasets as well as a set of standardized methods for transforming them between different coordinate systems and contextualizing novel findings within the broader literature.

Given the proliferation of such datasets in recent years, a large body of work has arisen focused on investigating similarities across brain maps [7, 18, 24, 31, 67, 80, 84]. Indeed, researchers have observed significant concordance in the spatial topology of brain maps derived from a wide variety of phenotypes, suggesting that these maps may reflect a fundamental organizational principle of the human brain. We reproduce this phenomenon in our example analyses, wherein twenty datasets provided with the neuromaps toolbox seem to group into two distinct clusters (Fig. 4). One cluster contains maps including the $\mathrm{T} 1 \mathrm{w} / \mathrm{T} 2 \mathrm{w}$ ratio [26], the principal component of gene expression [13, 47], cerebral blood flow, and metabolic glucose uptake [72], whereas the other is composed of maps like the principal functional gradient [46], intersubject functional variability [51], and developmental and evolutionary expansion [34]. In both cases, these clusters recapitulate a number of associations previously reported in the literature [13, 26, 31, 67].

Functionality for easing the computation of and standardizing such comparisons in the future is necessary to ensure that new datasets can be integrated into our broader understanding of the human brain. The neuromaps toolbox offers just such functionality, including workflows to transform, compare, and contextualize brain maps. The neuromaps distribution contains high-quality, group-level transformations between four standard coordinate systems, uniform interfaces for comparing brain maps, and access to nine null models for use in generating statistical inferences. By develop- 
ing the toolbox openly on GitHub (https://github.com/ netneurolab/neuromaps), it is our hope that neuromaps can serve as a community tool for broad use in brain mapping research moving forward.

One consideration researchers must be aware of when using the neuromaps toolbox is that the provided transformations between coordinate systems are meant to be applied to group-level data; however, in general, when subject-level data are available it is better to reprocess them in the desired coordinate system rather than transforming group-level aggregate data. Unfortunately, in practice, subject-level data for many commonly-used brain maps are not available to researchers, and so having high-quality transformations between systems is critical to ensuring that analyses are performed in the most accurate manner possible. We have based the provided transformations on state-of-the-art frameworks (i.e., registration fusion and multimodal surface matching) which have been rigorously assessed and validated on other datasets $[62,63,90]$. Moving forward, as new frameworks arise for mapping between coordinate systems we will endeavor to provide updated transformations when possible.

Altogether, the current report introduces a new opensource Python package, neuromaps, for use in human brain mapping research. The toolbox offers researchers access to a wide repository of brain maps taken from the published literature, high-quality transformations between four standard coordinate systems, and uniform interfaces for statistical comparisons between brain maps. As the rate at which new brain maps are generated in the field continues to grow, we hope that neuromaps will provide researchers a set of standardized workflows for better understanding what these data can tell us about the human brain.

\section{ACKNOWLEDGEMENTS}

This research was undertaken thanks in part to funding from the Canada First Research Excellence Fund, awarded to McGill University for the Healthy Brains for Healthy Lives initiative. This work was also supported in part by funding provided by Brain Canada, in partnership with Health Canada, for the Canadian Open Neuroscience Platform initiative. RDM acknowledges support from the Fonds du Recherche Québec - Nature et Technologies and the Canadian Open Neuroscience Platform. JYH acknowledges support from the Helmholtz International BigBrain Analytics \& Learning Laboratory, the Natural Sciences and Engineering Research Council of Canada, and the Fonds du ResercheQuébec - Nature et Technologies. SB acknowledges support from the NIH (R01 EB026299), a Discovery Grant from the Natural Science and Engineering Research Council of Canada (436355-13), the CIHR Canada Research Chair in Neural Dynamics of Brain Systems. TDS acknowledges support from the NIH (R01 MH112847 and R01 MH120482). MMC acknowledges support from the Natural Sciences and Engineering Research Council of Canada, the Canada Research Chairs Program, Healthy Brains for Healthy Lives, and the Fonds du Recherche Québec - Nature et Technologies. BM acknowledges support from the Natural Sciences and Engineering Research Council of Canada (NSERC Discovery Grant RGPIN \#017-04265) and from the Canada Research Chairs Program.
[1] Abraham, A., Pedregosa, F., Eickenberg, M., Gervais, P., Mueller, A., Kossaifi, J., Gramfort, A., Thirion, B., and Varoquaux, G. (2014). Machine learning for neuroimaging with scikit-learn. Frontiers in neuroinformatics, 8:14.

[2] Ad-Dab'bagh, Y., Lyttelton, O., Muehlboeck, J., Lepage, C., Einarson, D., Mok, K., Ivanov, O., Vincent, R., Lerch, J., Fombonne, E., et al. (2006). The CIVET image-processing environment: a fully automated comprehensive pipeline for anatomical neuroimaging research. In Proceedings of the 12th Annual Meeting of the Organization for Human Brain Mapping, volume 2266. Florence, Italy.

[3] Akbarian, S., Liu, C., Knowles, J. A., Vaccarino, F. M., Farnham, P. J., Crawford, G. E., Jaffe, A. E., Pinto, D., Dracheva, S., Geschwind, D. H., Mill, J., Nairn, A. C., Abyzov, A., Pochareddy, S., Prabhakar, S., Weissman, S., Sullivan, P. F., State, M. W., Weng, Z., Peters, M. A., White, K. P., Gerstein, M. B., Amiri, A., Armoskus, C., Ashley-Koch, A. E., Bae, T., Beckel-Mitchener, A., Berman, B. P., Coetzee, G. A., Coppola, G., Francoeur, N., Fromer, M., Gao, R., Grennan, K., Herstein, J., Kavanagh, D. H., Ivanov, N. A., Jiang, Y., Kitchen, R. R., Kozlenkov, A., Kundakovic, M., Li, M., Li, Z., Liu, S., Mangravite, L. M., Mat- tei, E., Markenscoff-Papadimitriou, E., Navarro, F. C. P., North, N., Omberg, L., Panchision, D., Parikshak, N., Poschmann, J., Price, A. J., Purcaro, M., Reddy, T. E., Roussos, P., Schreiner, S., Scuderi, S., Sebra, R., Shibata, M., Shieh, A. W., Skarica, M., Sun, W., Swarup, V., Thomas, A., Tsuji, J., van Bakel, H., Wang, D., Wang, Y., Wang, K., Werling, D. M., Willsey, A. J., Witt, H., Won, H., Wong, C. C. Y., Wray, G. A., Wu, E. Y., Xu, X., Yao, L., Senthil, G., Lehner, T., Sklar, P., and Sestan, N. (2015). The PsychENCODE project. Nature Neuroscience, 18(12):1707-1712.

[4] Alexander-Bloch, A. F., Shou, H., Liu, S., Satterthwaite, T. D., Glahn, D. C., Shinohara, R. T., Vandekar, S. N., and Raznahan, A. (2018). On testing for spatial correspondence between maps of human brain structure and function. NeuroImage, 178:540-551.

[5] Ashburner, J. and Friston, K. J. (1999). Nonlinear spatial normalization using basis functions. Human Brain Mapping, 7(4):254-266.

[6] Baryshnikova, A. (2016). Systematic functional annotation and visualization of biological networks. Cell systems, 2(6):412-421. 
[7] Baum, G. L., Cui, Z., Roalf, D. R., Ciric, R., Betzel, R. F., Larsen, B., Cieslak, M., Cook, P. A., Xia, C. H., Moore, T. M., Ruparel, K., Oathes, D. J., Alexander-Bloch, A. F., Shinohara, R. T., Raznahan, A., Gur, R. E., Gur, R. C., Bassett, D. S., and Satterthwaite, T. D. (2020). Development of structure-function coupling in human brain networks during youth. Proceedings of the National Academy of Sciences, 117(1):771-778.

[8] Beliveau, V., Ganz, M., Feng, L., Ozenne, B., Højgaard, L., Fisher, P. M., Svarer, C., Greve, D. N., and Knudsen, G. M. (2017). A high-resolution in vivo atlas of the human brain's serotonin system. Journal of Neuroscience, 37(1):120-128.

[9] Bethlehem, R. A., Seidlitz, J., White, S. R., Vogel, J. W., Anderson, K. M., Adamson, C., Adler, S., Alexopoulos, G. S., Anagnostou, E., Areces-Gonzalez, A., et al. (2021). Brain charts for the human lifespan. bioRxiv.

[10] Brett, M., Johnsrude, I. S., and Owen, A. M. (2002). The problem of functional localization in the human brain. Nature Reviews Neuroscience, 3(3):243-249.

[11] Brett, M., Markiewicz, C. J., Hanke, M., Côté, M.-A., Cipollini, B., McCarthy, P., Cheng, C. P., Halchenko, Y. O., Cottaar, M., Ghosh, S., and et al. (2019). nipy/nibabel. Zenodo, doi:10.5281/zenodo.591597.

[12] Buckner, R. L., Krienen, F. M., Castellanos, A., Diaz, J. C., and Yeo, B. T. (2011). The organization of the human cerebellum estimated by intrinsic functional connectivity. Journal of Neurophysiology, 106(5):2322-2345.

[13] Burt, J. B., Demirtaş, M., Eckner, W. J., Navejar, N. M., Ji, J. L., Martin, W. J., Bernacchia, A., Anticevic, A., and Murray, J. D. (2018). Hierarchy of transcriptomic specialization across human cortex captured by structural neuroimaging topography. Nature neuroscience, 21(9):12511259.

[14] Burt, J. B., Helmer, M., Shinn, M., Anticevic, A., and Murray, J. D. (2020). Generative modeling of brain maps with spatial autocorrelation. NeuroImage.

[15] Collins, D. L., Neelin, P., Peters, T. M., and Evans, A. C. (1994). Automatic 3D intersubject registration of MR volumetric data in standardized Talairach space. Journal of Computer Assisted Tomography, 18(2):192-205.

[16] Cornblath, E. J., Ashourvan, A., Kim, J. Z., Betzel, R. F., Ciric, R., Adebimpe, A., Baum, G. L., He, X., Ruparel, K., Moore, T. M., Gur, R. C., Gur, R. E., Shinohara, R. T., Roalf, D. R., Satterthwaite, T. D., and Bassett, D. S. (2020). Temporal sequences of brain activity at rest are constrained by white matter structure and modulated by cognitive demands. Communications Biology, 3(1):1-12.

[17] Dale, A. M., Fischl, B., and Sereno, M. I. (1999). Cortical surface-based analysis: I. Segmentation and surface reconstruction. NeuroImage, 9(2):179-194.

[18] Demirtaş, M., Burt, J. B., Helmer, M., Ji, J. L., Adkinson, B. D., Glasser, M. F., Van Essen, D. C., Sotiropoulos, S. N., Anticevic, A., and Murray, J. D. (2019). Hierarchical heterogeneity across human cortex shapes large-scale neural dynamics. Neuron, 101(6):1181-1194.

[19] Evans, A. C., Collins, D. L., Mills, S., Brown, E. D., Kelly, R. L., and Peters, T. M. (1993). 3d statistical neuroanatomical models from 305 MRI volumes. In 1993 IEEE conference record nuclear science symposium and medical imaging conference, pages 1813-1817. IEEE.

[20] Fischl, B., Sereno, M. I., and Dale, A. M. (1999a). Cortical surface-based analysis: II: inflation, flattening, and a surface-based coordinate system. NeuroImage, 9(2):195-
207.

[21] Fischl, B., Sereno, M. I., Tootell, R. B., and Dale, A. M. (1999b). High-resolution intersubject averaging and a coordinate system for the cortical surface. Human Brain Mapping, 8(4):272-284.

[22] Fonov, V., Evans, A. C., Botteron, K., Almli, C. R., McKinstry, R. C., Collins, D. L., Group, B. D. C., et al. (2011). Unbiased average age-appropriate atlases for pediatric studies. NeuroImage, 54(1):313-327.

[23] Fonov, V. S., Evans, A. C., McKinstry, R. C., Almli, C., and Collins, D. (2009). Unbiased nonlinear average ageappropriate brain templates from birth to adulthood. NeuroImage, 47:S102.

[24] Gao, R., van den Brink, R. L., Pfeffer, T., and Voytek, B. (2020). Neuronal timescales are functionally dynamic and shaped by cortical microarchitecture. Elife, 9:e61277.

[25] Glasser, M. F., Coalson, T. S., Robinson, E. C., Hacker, C. D., Harwell, J., Yacoub, E., Ugurbil, K., Andersson, J., Beckmann, C. F., Jenkinson, M., et al. (2016). A multimodal parcellation of human cerebral cortex. Nature, 536(7615):171-178.

[26] Glasser, M. F., Goyal, M. S., Preuss, T. M., Raichle, M. E., and Van Essen, D. C. (2014). Trends and properties of human cerebral cortex: correlations with cortical myelin content. NeuroImage, 93:165-175.

[27] Glasser, M. F., Sotiropoulos, S. N., Wilson, J. A., Coalson, T. S., Fischl, B., Andersson, J. L., Xu, J., Jbabdi, S., Webster, M., Polimeni, J. R., et al. (2013). The minimal preprocessing pipelines for the Human Connectome Project. NeuroImage, 80:105-124.

[28] Glasser, M. F. and Van Essen, D. C. (2011). Mapping human cortical areas in vivo based on myelin content as revealed by T1-and T2-weighted MRI. Journal of Neuroscience, 31(32):11597-11616.

[29] Gorgolewski, K. J., Varoquaux, G., Rivera, G., Schwartz, Y., Sochat, V. V., Ghosh, S. S., Maumet, C., Nichols, T. E., Poline, J.-B., Yarkoni, T., et al. (2016). NeuroVault.org: Arepository for sharing unthresholded statistical maps, parcellations, and atlases of the human brain. NeuroImage, 124:1242-1244.

[30] Hamm, J., Ye, D. H., Verma, R., and Davatzikos, C. (2010). GRAM: A framework for geodesic registration on anatomical manifolds. Medical Image Analysis, 14(5):633-642.

[31] Hansen, J. Y., Markello, R. D., Vogel, J. W., Seidlitz, J., Bzdok, D., and Misic, B. (2021a). Mapping gene transcription and neurocognition across human neocortex. Nature Human Behaviour, pages 1-11.

[32] Hansen, J. Y., Shafiei, G., Markello, R. D., Smart, K., Cox, S., Wu, Y., Gallezot, J.-D., Étienne, A., Servaes, S., Scala, S. G., DuBois, J. M., Wainstein, G., Bezgin, G., Funck, T., Schmitz, T. W., Spreng, R. N., Soucy, J.-P., Baillet, S., Guimond, S., Hietala, J., Bédard, M.-A., Leyton, M., Kobayashi, E., Rosa-Neto, P., Palomero-Gallagher, N., Shine, J. M., Carson, R. E., Tuominen, L., Dagher, A., and Misic, B. (2021b). Mapping neurotransmitter systems to the structural and functional organization of the human neocortex. bioRxiv.

[33] Hawrylycz, M., Lein, E. S., Guillozet-Bongaarts, A. L., Shen, E. H., Ng, L., Miller, J. A., Van De Lagemaat, L. N., Smith, K. A., Ebbert, A., Riley, Z. L., et al. (2012). An anatomically comprehensive atlas of the adult human brain transcriptome. Nature, 489(7416):391. 
[34] Hill, J., Inder, T., Neil, J., Dierker, D., Harwell, J., and Van Essen, D. (2010). Similar patterns of cortical expansion during human development and evolution. Proceedings of the National Academy of Sciences, 107(29):1313513140.

[35] Holmes, A. J., Hollinshead, M. O., O'keefe, T. M., Petrov, V. I., Fariello, G. R., Wald, L. L., Fischl, B., Rosen, B. R., Mair, R. W., Roffman, J. L., et al. (2015). Brain Genomics Superstruct Project initial data release with structural, functional, and behavioral measures. Scientific data, 2(1):1-16.

[36] Huntenburg, J. M., Bazin, P.-L., Goulas, A., Tardif, C. L., Villringer, A., and Margulies, D. S. (2017). A systematic relationship between functional connectivity and intracortical myelin in the human cerebral cortex. Cerebral Cortex, 27(2):981-997.

[37] Huntenburg, J. M., Bazin, P.-L., and Margulies, D. S. (2018). Large-scale gradients in human cortical organization. Trends Cogn Sci, 22(1):21-31.

[38] Hunter, J. D. (2007). Matplotlib: A 2D graphics environment. Computing in Science \& Engineering, 9(3):90-95.

[39] Jenkinson, M., Beckmann, C. F., Behrens, T. E., Woolrich, M. W., and Smith, S. M. (2012). FSL. NeuroImage, 62(2):782-790.

[40] Kantonen, T., Karjalainen, T., Isojärvi, J., Nuutila, P., Tuisku, J., Rinne, J., Hietala, J., Kaasinen, V., Kalliokoski, K., Scheinin, H., et al. (2020). Interindividual variability and lateralization of $\mu$-opioid receptors in the human brain. NeuroImage, 217:116922.

[41] Kluyver, T., Ragan-Kelley, B., Pérez, F., Granger, B. E., Bussonnier, M., Frederic, J., Kelley, K., Hamrick, J. B., Grout, J., Corlay, S., et al. (2016). Jupyter Notebooks-A publishing format for reproducible computational workflows. In Loizides, F. and Scmidt, B., editors, Positioning and Power in Academic Publishing: Players, Agents and Agendas, pages 87-90. IOS Press.

[42] Laird, A. R., Robinson, J. L., McMillan, K. M., TordesillasGutiérrez, D., Moran, S. T., Gonzales, S. M., Ray, K. L., Franklin, C., Glahn, D. C., Fox, P. T., et al. (2010). Comparison of the disparity between Talairach and MNI coordinates in functional neuroimaging data: validation of the Lancaster transform. NeuroImage, 51(2):677-683.

[43] Lancaster, J. L., Tordesillas-Gutiérrez, D., Martinez, M., Salinas, F., Evans, A., Zilles, K., Mazziotta, J. C., and Fox, P. T. (2007). Bias between MNI and Talairach coordinates analyzed using the ICBM-152 brain template. $\mathrm{Hu}$ man Brain Mapping, 28(11):1194-1205.

[44] Li, X., Ai, L., Giavasis, S., Jin, H., Feczko, E., Xu, T., Clucas, J., Franco, A., Heinsfeld, A. S., Adebimpe, A., et al. (2021). Moving beyond processing and analysis-related variation in neuroscience. bioRxiv.

[45] Marcus, D., Harwell, J., Olsen, T., Hodge, M., Glasser, M., Prior, F., Jenkinson, M., Laumann, T., Curtiss, S., and Van Essen, D. (2011). Informatics and data mining tools and strategies for the human connectome project. Frontiers in Neuroinformatics, 5:4.

[46] Margulies, D. S., Ghosh, S. S., Goulas, A., Falkiewicz, M., Huntenburg, J. M., Langs, G., Bezgin, G., Eickhoff, S. B., Castellanos, F. X., Petrides, M., Jeffries, E., and Smallwood, J. (2016). Situating the default-mode network along a principal gradient of macroscale cortical organization. Proceedings of the National Academy of Sciences, 113(44):12574-12579.
[47] Markello, R., Arnatkeviciute, A., Poline, J.-B., Fulcher, B. D., Fornito, A., and Misic, B. (2021). Standardizing workflows in imaging transcriptomics with the abagen toolbox. bioRxiv.

[48] Markello, R. D. and Misic, B. (2021). Comparing spatial null models for brain maps. NeuroImage, page 118052.

[49] Mazziotta, J., Toga, A., Evans, A., Fox, P., Lancaster, J., Zilles, K., Woods, R., Paus, T., Simpson, G., Pike, B., et al. (2001). A probabilistic atlas and reference system for the human brain: International Consortium for Brain Mapping (ICBM). Philosophical Transactions of the Royal Society of London. Series B: Biological Sciences, 356(1412):1293-1322.

[50] McKinney, W. et al. (2010). Data structures for statistical computing in Python. In Proceedings of the 9th Python in Science Conference, volume 445, pages 51-56. Austin, TX.

[51] Mueller, S., Wang, D., Fox, M. D., Yeo, B. T., Sepulcre, J., Sabuncu, M. R., Shafee, R., Lu, J., and Liu, H. (2013). Individual variability in functional connectivity architecture of the human brain. Neuron, 77(3):586-595.

[52] Murray, J. D., Bernacchia, A., Freedman, D. J., Romo, R., Wallis, J. D., Cai, X., Padoa-Schioppa, C., Pasternak, T., Seo, H., Lee, D., and Wang, X.-J. (2014). A hierarchy of intrinsic timescales across primate cortex. Nature Neuroscience, 17(12):1661-1663.

[53] Naganawa, M., Nabulsi, N., Henry, S., Matuskey, D., Lin, S.-F., Slieker, L., Schwarz, A. J., Kant, N., Jesudason, C., Ruley, K., et al. (2021). First-in-human assessment of 11clsn3172176, an $\mathrm{m} 1$ muscarinic acetylcholine receptor pet radiotracer. Journal of Nuclear Medicine, 62(4):553-560.

[54] Nenning, K.-H., Liu, H., Ghosh, S. S., Sabuncu, M. R., Schwartz, E., and Langs, G. (2017). Diffeomorphic functional brain surface alignment: Functional demons. NeuroImage, 156:456-465.

[55] Nørgaard, M., Beliveau, V., Ganz, M., Svarer, C., Pinborg, L. H., Keller, S. H., Jensen, P. S., Greve, D. N., and Knudsen, G. M. (2021). A high-resolution in vivo atlas of the human brain's benzodiazepine binding site of $\mathrm{GABA}_{A}$ receptors. NeuroImage, 232:117878.

[56] Oliphant, T. E. (2006). A guide to NumPy, volume 1. Trelgol Publishing USA.

[57] Paquola, C., Royer, J., Lewis, L. B., Lepage, C., Glatard, T., Wagstyl, K., DeKraker, J., Toussaint, P.-J., Valk, S. L., Collins, D. L., et al. (2021). BigBrainWarp: Toolbox for integration of BigBrain 3D histology with mutlimodal neuroimaging. bioRxiv.

[58] Pedregosa, F., Varoquaux, G., Gramfort, A., Michel, V., Thirion, B., Grisel, O., Blondel, M., Prettenhofer, P., Weiss, R., Dubourg, V., et al. (2011). Scikit-learn: Machine learning in Python. Journal of Machine Learning Research, 12 (Oct):2825-2830.

[59] Pérez, F. and Granger, B. E. (2007). IPython: A system for interactive scientific computing. Computing in Science \& Engineering, 9(3):21-29.

[60] Reardon, P., Seidlitz, J., Vandekar, S., Liu, S., Patel, R., Park, M. T. M., Alexander-Bloch, A., Clasen, L. S., Blumenthal, J. D., Lalonde, F. M., et al. (2018). Normative brain size variation and brain shape diversity in humans. Science, 360(6394):1222-1227.

[61] Reimand, J., Isserlin, R., Voisin, V., Kucera, M., TannusLopes, C., Rostamianfar, A., Wadi, L., Meyer, M., Wong, J., Xu, C., et al. (2019). Pathway enrichment analysis and visualization of omics data using g: Profiler, gsea, cytoscape and enrichmentmap. Nature protocols, 14(2):482-517. 
[62] Robinson, E. C., Garcia, K., Glasser, M. F., Chen, Z., Coalson, T. S., Makropoulos, A., Bozek, J., Wright, R., Schuh, A., Webster, M., et al. (2018). Multimodal surface matching with higher-order smoothness constraints. NeuroImage, 167:453-465.

[63] Robinson, E. C., Jbabdi, S., Glasser, M. F., Andersson, J., Burgess, G. C., Harms, M. P., Smith, S. M., Van Essen, D. C., and Jenkinson, M. (2014). MSM: a new flexible framework for Multimodal Surface Matching. NeuroImage, 100:414-426.

[64] Rueckert, D., Sonoda, L. I., Hayes, C., Hill, D. L., Leach, M. O., and Hawkes, D. J. (1999). Nonrigid registration using free-form deformations: application to breast MR images. IEEE transactions on medical imaging, 18(8):712721.

[65] Savli, M., Bauer, A., Mitterhauser, M., Ding, Y.-S., Hahn, A., Kroll, T., Neumeister, A., Haeusler, D., Ungersboeck, J., Henry, S., et al. (2012). Normative database of the serotonergic system in healthy subjects using multi-tracer pet. Neuroimage, 63(1):447-459.

[66] Scholtens, L. H., de Reus, M. A., de Lange, S. C., Schmidt, R., and van den Heuvel, M. P. (2018). An MRI Von Economo-Koskinas atlas. NeuroImage, 170:249-256.

[67] Shafiei, G., Markello, R. D., De Wael, R. V., Bernhardt, B. C., Fulcher, B. D., and Misic, B. (2020). Topographic gradients of intrinsic dynamics across neocortex. Elife, 9:e62116.

[68] Smart, K., Cox, S. M., Scala, S. G., Tippler, M., Jaworska, N., Boivin, M., Séguin, J. R., Benkelfat, C., and Leyton, M. (2019). Sex differences in [11 c] abp688 binding: a positron emission tomography study of mglu5 receptors. European journal of nuclear medicine and molecular imaging, 46(5):1179-1183.

[69] Sydnor, V. J., Larsen, B., Bassett, D. S., Alexander-Bloch, A., Fair, D. A., Liston, C., Mackey, A. P., Milham, M. P., Pines, A., Roalf, D. R., et al. (2021). Neurodevelopment of the association cortices: Patterns, mechanisms, and implications for psychopathology. Neuron.

[70] Tadel, F., Baillet, S., Mosher, J. C., Pantazis, D., and Leahy, R. M. (2011). Brainstorm: a user-friendly application for $\mathrm{meg} / \mathrm{eeg}$ analysis. Computational intelligence and neuroscience, 2011.

[71] Tong, T., Aganj, I., Ge, T., Polimeni, J. R., and Fischl, B. (2017). Functional density and edge maps: Characterizing functional architecture in individuals and improving cross-subject registration. NeuroImage, 158:346-355.

[72] Vaishnavi, S. N., Vlassenko, A. G., Rundle, M. M., Snyder, A. Z., Mintun, M. A., and Raichle, M. E. (2010). Regional aerobic glycolysis in the human brain. Proceedings of the National Academy of Sciences, 107(41):17757-17762.

[73] Van Der Walt, S., Colbert, S. C., and Varoquaux, G. (2011). The NumPy array: a structure for efficient numerical computation. Computing in Science \& Engineering, 13(2):22.

[74] Van Essen, D. C. (2002). Windows on the brain: the emerging role of atlases and databases in neuroscience. Current Opinion in Neurobiology, 12(5):574-579.

[75] Van Essen, D. C. (2005). A population-average, landmarkand surface-based (PALS) atlas of human cerebral cortex. NeuroImage, 28(3):635-662.

[76] Van Essen, D. C., Glasser, M. F., Dierker, D. L., Harwell, J., and Coalson, T. (2012). Parcellations and hemispheric asymmetries of human cerebral cortex analyzed on surface-based atlases. Cerebral Cortex, 22(10):2241-
2262.

[77] Van Essen, D. C., Smith, J., Glasser, M. F., Elam, J., Donahue, C. J., Dierker, D. L., Reid, E. K., Coalson, T., and Harwell, J. (2017). The brain analysis library of spatial maps and atlases (BALSA) database. NeuroImage, 144:270-274.

[78] Van Essen, D. C., Smith, S. M., Barch, D. M., Behrens, T. E., Yacoub, E., Ugurbil, K., Consortium, W.-M. H., et al. (2013). The WU-Minn human connectome project: an overview. NeuroImage, 80:62-79.

[79] Váša, F., Seidlitz, J., Romero-Garcia, R., Whitaker, K. J., Rosenthal, G., Vértes, P. E., Shinn, M., Alexander-Bloch, A., Fonagy, P., Dolan, R. J., Jones, P. B., Goodyer, I. M., the NSPN consortium, Sporns, O., and Bullmore, E. T. (2018). Adolescent tuning of association cortex in human structural brain networks. Cerebral Cortex, 28(1):281294.

[80] Vázquez-Rodríguez, B., Suárez, L. E., Markello, R. D., Shafiei, G., Paquola, C., Hagmann, P., Van Den Heuvel, M. P., Bernhardt, B. C., Spreng, R. N., and Mišić, B. (2019). Gradients of structure-function tethering across neocortex. Proceedings of the National Academy of Sciences, 116(42):21219-21227.

[81] Virtanen, P., Gommers, R., Oliphant, T. E., Haberland, M., Reddy, T., Cournapeau, D., Burovski, E., Peterson, P., Weckesser, W., Bright, J., van der Walt, S. J., Brett, M., Wilson, J., Millman, K. J., Mayorov, N., Nelson, A. R. J., Jones, E., Kern, R., Larson, E., Carey, C. J., Illhan Polat, Feng, Y., Moore, E. W., VanderPlas, J., Laxalde, D., Perktold, J., Cimrman, R., Henriksen, I., Quintero, E. A., Harris, C. R., Archibald, A. M., Ribeiro, A. H., Pedregosa, F., van Mulbregt, P., and the SciPy 1.0 Contributors (2020). Scipy 1.0: fundamental algorithms for scientific computing in python. Nature Methods, pages 1-12.

[82] Vos de Wael, R., Benkarim, O., Paquola, C., Lariviere, S., Royer, J., Tavakol, S., Xu, T., Hong, S.-J., Langs, G., Valk, S., Mišić, B., Milham, M., Margulies, D., Smallwood, J., and Bernhardt, B. C. (2020). BrainSpace: a toolbox for the analysis of macroscale gradients in neuroimaging and connectomics datasets. Communications Biology, 3(1):110.

[83] Wagstyl, K., Larocque, S., Cucurull, G., Lepage, C., Cohen, J. P., Bludau, S., Palomero-Gallagher, N., Lewis, L. B., Funck, T., Spitzer, H., et al. (2020). Bigbrain 3d atlas of cortical layers: Cortical and laminar thickness gradients diverge in sensory and motor cortices. PLoS Biol, 18(4):e3000678.

[84] Wang, P., Kong, R., Kong, X., Liégeois, R., Orban, C., Deco, G., Van Den Heuvel, M. P., and Yeo, B. T. (2019). Inversion of a large-scale circuit model reveals a cortical hierarchy in the dynamic resting human brain. Science Advances, 5(1):eaat7854.

[85] Waskom, M., Botvinnik, O., Ostblom, J., Gelbart, M., Lukauskas, S., Hobson, P., Gemperline, D. C., Augspurger, T., Halchenko, Y., Cole, J. B., Warmenhoven, J., de Ruiter, J., Pye, C., Hoyer, S., Vanderplas, J., Villalba, S., Kunter, G., Quintero, E., Bachant, P., Martin, M., Meyer, K., Swain, C., Miles, A., Brunner, T., O'Kane, D., Yarkoni, T., Williams, M. L., Evans, C., and Fitzgerald, C. (2020a). mwaskom/seaborn. Zenodo, doi:10.5281/zenodo.592845.

[86] Waskom, M., Larson, E., Brodbeck, C., Gramfort, A., Burns, S., Luessi, M., Weidemann, C. T., Bitzer, S., Markiewicz, C., LaPlante, R., Halchenko, Y., Engemann, 
D. A., van Vliet, M., Ghosh, S., Klein, N., Piantoni, G., Brett, M., Gwilliams, L., King, J.-R., and Liu, D. (2020b). nipy/pysurfer. Zenodo, doi:10.5281/zenodo.592515.

[87] Wei, Y., de Lange, S. C., Scholtens, L. H., Watanabe, K., Ardesch, D. J., Jansen, P. R., Savage, J. E., Li, L., Preuss, T. M., Rilling, J. K., et al. (2019). Genetic mapping and evolutionary analysis of human-expanded cognitive networks. Nature Communications, 10(1):1-11.

[88] Whitaker, K. J., Vértes, P. E., Romero-Garcia, R., Váša, F., Moutoussis, M., Prabhu, G., Weiskopf, N., Callaghan, M. F., Wagstyl, K., Rittman, T., Tait, R., Ooi, C., Suckling, J., Inkster, B., Fonagy, P., Dolan, R. J., Jones, P. B., Goodyer, I. M., the NSPN Consortium, and Bullmore, E. T. (2016). Adolescence is associated with genomically patterned consolidation of the hubs of the human brain connectome. Proceedings of the National Academy of Sciences, 113(32):9105-9110.

[89] Woods, R. P., Grafton, S. T., Holmes, C. J., Cherry, S. R., and Mazziotta, J. C. (1998). Automated image registration: I. General methods and intrasubject, intramodality validation. Journal of Computer Assisted Tomography, 22(1):139-152.

[90] Wu, J., Ngo, G. H., Greve, D., Li, J., He, T., Fischl, B., Eickhoff, S. B., and Yeo, B. T. (2018). Accurate nonlinear mapping between MNI volumetric and FreeSurfer surface coordinate systems. Human Brain Mapping, 39(9):37933808.

[91] Xu, T., Nenning, K.-H., Schwartz, E., Hong, S.-J., Vogelstein, J. T., Goulas, A., Fair, D. A., Schroeder, C. E., Margulies, D. S., Smallwood, J., et al. (2020). Crossspecies functional alignment reveals evolutionary hierarchy within the connectome. NeuroImage, 223:117346.

[92] Yarkoni, T., Poldrack, R. A., Nichols, T. E., Van Essen, D. C., and Wager, T. D. (2011). Large-scale automated synthesis of human functional neuroimaging data. Nature Methods, 8(8):665.

[93] Yushkevich, P. A., Wang, H., Pluta, J., and Avants, B. B. (2012). From label fusion to correspondence fusion: a new approach to unbiased groupwise registration. In 2012 IEEE Conference on Computer Vision and Pattern Recognition, pages 956-963. IEEE.

[94] Zilles, K. and Palomero-Gallagher, N. (2017). Multiple transmitter receptors in regions and layers of the human cerebral cortex. Frontiers in neuroanatomy, 11:78. 
Table S1. Standard coordinate system files available in neuromaps

\begin{tabular}{lll}
\hline Coordinate system & Density / resolution & Provided files \\
\hline fsLR & $4 \mathrm{k}$ & midthickness, inflated, sphere \\
fsLR & $8 \mathrm{k}$ & midthickness, inflated, sphere \\
fsLR & $32 \mathrm{k}$ & midthickness, inflated, very inflated, sphere \\
fsLR & $164 \mathrm{k}$ & midthickness, inflated, very inflated, sphere \\
fsaverage & $3 \mathrm{k}$ & white, pial, inflated, sphere \\
fsaverage & $10 \mathrm{k}$ & white, pial, inflated, sphere \\
fsaverage & $41 \mathrm{k}$ & white, pial, inflated, sphere \\
fsaverage & $164 \mathrm{k}$ & white, pial, inflated, sphere \\
CIVET & $41 \mathrm{k}$ & white, midthickness, inflated, very inflated, sphere \\
MNI152 & $1 \mathrm{~mm}$ & T1w, T2w, PD, brainmask \\
MNI152 & $2 \mathrm{~mm}$ & T1w, T2w, PD, brainmask \\
MNI152 & $3 \mathrm{~mm}$ & T1w, T2w, PD, brainmask
\end{tabular}

Table S2. Published brain maps available in neuromaps

\begin{tabular}{llcc}
\hline Map name & Coordinate system & Density & Citation \\
\hline Myelin map & fsLR & $32 \mathrm{k}$ & Glasser et al. [25] \\
Cortical thickness & fsLR & $32 \mathrm{k}$ & Glasser et al. [25] \\
Allen Human Brain Atlas PC1 & fsaverage & $10 \mathrm{k}$ & Markello et al. [47] \\
Delta power & fsLR & $4 \mathrm{k}$ & Van Essen et al. [78] \\
Theta power & fsLR & $4 \mathrm{k}$ & Van Essen et al. [78] \\
Alpha power & fsLR & $4 \mathrm{k}$ & Van Essen et al. [78] \\
Beta power & fsLR & $4 \mathrm{k}$ & Van Essen et al. [78] \\
Low gamma power & fsLR & $4 \mathrm{k}$ & Van Essen et al. [78] \\
High gamma power & fsLR & $4 \mathrm{k}$ & Van Essen et al. [78] \\
Intrinsic timescale & fsLR & $4 \mathrm{k}$ & Van Essen et al. [78] \\
Developmental expansion & fsLR & $164 \mathrm{k}$ & Hill et al. [34] \\
Evolutionary expansion & fsLR & $164 \mathrm{k}$ & Hill et al. [34] \\
Functional gradient & fsLR & $32 \mathrm{k}$ & Margulies et al. [46] \\
Inter-subject functional variability & fsaverage & $1 \mathrm{k}$ & Mueller et al. [51] \\
NeuroSynth PC1 & MNI152 & $2 \mathrm{~mm}$ & Yarkoni et al. [92] \\
Cerebral blood flow (CBF) & fsLR & $164 \mathrm{k}$ & Vaishnavi et al. [72] \\
Cerebral blood volume (CBV) & fsLR & $164 \mathrm{k}$ & Vaishnavi et al. [72] \\
Oxygen metabolism (CMRO2) & fsLR & $164 \mathrm{k}$ & Vaishnavi et al. [72] \\
Glucose metabolism (GMRGlu) & fsLR & $164 \mathrm{k}$ & Vaishnavi et al. [72] \\
Allometric scaling (PNC) & CIVET & $41 \mathrm{k}$ & Reardon et al. [60] \\
Allometric scaling (NIH) & CIVET & $41 \mathrm{k}$ & Reardon et al. [60] \\
Neurotransmitter receptors/transporters & MNI152 & - & Hansen et al. [32]
\end{tabular}

